\title{
Aplicação de métricas socioespaciais para caracterização de divisões administrativas municipais: Estudo de caso para o Município de São Paulo
}

\section{Application of socio-spatial metrics for the characterization of municipal administrative divisions: Case study for the Municipality of São Paulo}

\author{
Cruz, Rafael Barreto Castelo da'; Marins, Karin Regina de Castro². \\ ' Escola Politécnica da Universidade de São Paulo,Brasil, rafaelcastelo@usp.br' \\ 2Escola Politécnica da Universidade de São Paulo,Brasil, karin,marins@usp.br
}

\begin{abstract}
RESUMO
As métricas socioespaciais permitem analisar uma situação urbana e, de forma multicriterial, podem apontar resultados divergentes entre si. O objetivo deste trabalho é avaliar se existe uma relação de dependência entre parâmetros que caracterizam a forma urbana. A pesquisa exploratória, com apoio de uma breve revisão bibliográfica, apresenta a definição e a metodologia de cálculo do índice de compacidade, do índice de diversidade de uso, da densidade populacional e da densidade de empregos, com levantamento de dados oficiais para o município de São Paulo. Os resultados apontam, para o caso paulistano, que a relação entre as métricas sócio espaciais não é sistemática, ou seja, todos os melhores casos de compacidade, não estão associados aos melhores casos de diversidade, densidade humana e densidade de emprego. Não é clara e direta a dependência entre essas variáveis urbanas, sendo possivelmente influenciadas, em uma abordagem mais abrangente, por outros fatores urbanos. Por esta razão, ao se adotar métricas socioespaciais da forma urbana, no planejamento dos municípios, é importante ter uma visão crítica sobre o conjunto dos seus resultados e buscar ampliar a análise mediante identificação e análise de parâmetros complementares da morfologia urbana.
\end{abstract}

Palavras-chave: Indicadores Urbanos, Métricas socioespaciais, Forma Urbana, Planejamento Urbano.

\section{ABSTRACT}

Urban socio-spatial metrics allow an urban situation to be analyzed and, in a multi-criteria way, can show divergent results among them. Therefore, the objective of this work is to evaluate if there is a relation of dependence between parameters that characterize the urban form. The

${ }^{1}$ CRUZ, Rafael Barreto Castelo da; MARINS, Karin Regina de Castro. Aplicação de métricas socioespaciais para caracterização de divisões administrativas municipais: Estudo de caso para o Município de São Paulo. In: II SIMPÓSIO NACIONAL DE GESTÃO E ENGENHARIA URBANA: SINGEURB, 2019, São Paulo. Anais... Porto Alegre: ANTAC, 2019. 
research developed in an exploratory way, supported by a brief bibliographical review, presents the definition and the methodology of calculation of the index of compactness, the index of diversity of use, population density and the density of jobs, with documentary survey and data for the municipality of São Paulo. The results indicate that, in the case of São Paulo, the relationship between socio-spatial metrics is not systematic, that is, all the best cases of compactness, are not associated with the best cases of diversity, human density and employment density. It is not clear and direct the dependence between these urban variables, being possibly influenced, in a more comprehensive approach, by other urban factors. For this reason, when adopting socio-spatial metrics of the urban form, in the planning of the municipalities, it is important to have a critical view on all of its results and to seek to broaden the analysis through identification and analysis of complementary parameters of the urban morphology.

Keywords: Urban Indicators, Socio-spatial Metrics, Urban Form, Urban Planning.

\section{INTRODUÇÃO}

A urbanização ${ }^{2}$ é um fenômeno que pode analisado, no Brasil, verificando que a população urbana, em relação à população total, saltou de 31,3\% em 1940 para 84,3\% em 2010 (IBGE, 2010). Neste cenário as cidades enfrentarão novos desafios para atender este contingente de pessoas 3 . O marco legal para a formulação da Política Urbana brasileira vigente tem origem na Constituição Federal (CF) de 1988, em especial, em seus artigos 182 e 183, posteriormente regulamentados pelo Estatuto da Cidade (EC), Lei 10.257, de 2001, bem como pelo Estatuto da Metrópole, Lei 13.089, de 2015 e tem uma relação hierárquica, conforme a figura 1.

Figura 1 - Associação hierárquica de políticas urbanas

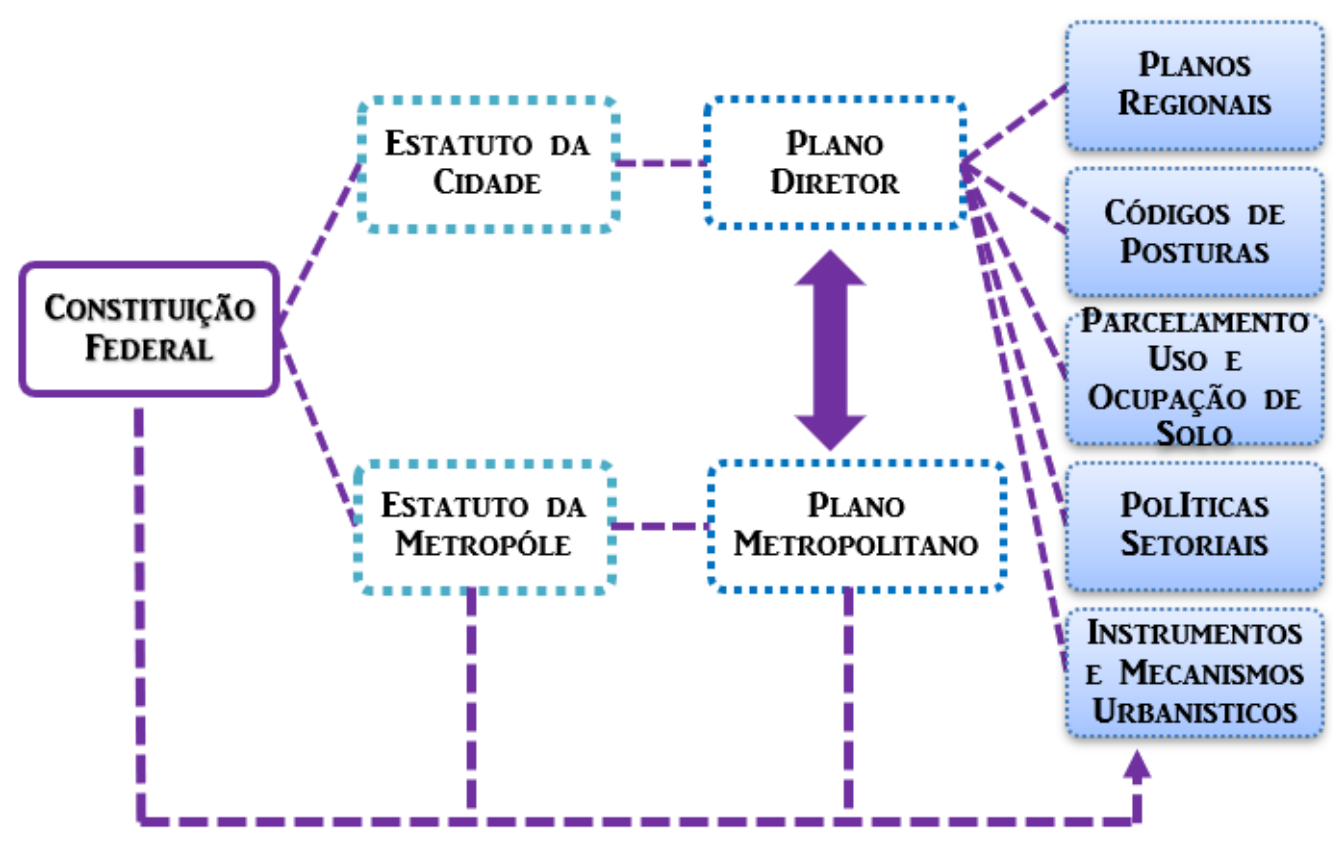

Fonte: Os Autores (2016) - Adaptado de Marins (2016) ${ }^{4}$

\footnotetext{
${ }^{2}$ Urbanização é o processo o qual a população ocupa predominante cidades.

${ }^{3}$ IBGE, censos de 1940, 1960, 1970 e 2000.

${ }^{4}$ Fluxograma com a legislação vigente para o desenvolvimento dos Planos Diretores apresenta também os principais artigos que influenciam nos aspectos de planejamento urbano nas cidades e áreas metropolitanas. Fonte: $\mathrm{O}$ autor adaptado de MARINS, K.R.C.C. Governança em áreas urbanas. Apresentação. PPGEC, 2016.
} 
Entende-se indicador como um instrumento que permite mensurar as modificações nas características de um sistema, de tal forma que seu enfoque integrador, forneça informações condensadas sobre vários aspectos de um sistema, apontam Masera; Astier; Lopez-Ridaura (2000). No caso do planejamento urbano. Alguns indicadores podem ser expressos por métricas socioespaciais.

O objetivo deste trabalho é avaliar a relação de dependência entre métricas sócio espaciais que caracterizam a forma urbana, tais como a compacidade, a diversidade de usos, a densidade populacional e a densidade de empregos, para as diferentes regiões administrativas do município de São Paulo.

Esta pesquisa exploratória baseia-se em breve revisão bibliográfica sobre a definição, e o cálculo dos índices de compacidade e diversidade de uso e das densidades populacional e de empregos, assim como dados geográficos oficiais das divisões oficiais adotadas no município de São Paulo.

\section{ANÁLISE BIBLIOGRÁFICA - MÉTRICAS DA FORMA URBANA}

O nível de desenvolvimento econômico e social de uma área urbana pode ser medido pela taxa de urbanização, pelo crescimento da população urbana, pela densidade populacional e pela densidade de empregos, aponta Zhen (2018).

Os indicadores espaciais permitem examinar questões de diversidade, tais como a distribuição e concentração de diferentes atividades em uma região, a diversidade no uso do solo com moradia, edifícios comerciais, área verdes e áreas não residenciais, e podem ser examinadas dentro dos limites de um distrito ou entre vários distritos (BOURDIC, SALAT \& NOWACKI, 2012).

Para avaliar a diversidade, é aplicado o índice de diversidade, dado pela equação 1, onde ni é a quantidade de ocupações de um tipo de uso da categoria i; e N é a quantidade total de unidades todos os tipos de uso conforme Bourdic, Salat e Nowacki (2012).

$$
\text { Indice de Diversidade }=I=1-\sum_{i=1}^{\infty}\left(\frac{n_{i}}{N}\right)^{2}
$$

É possivel descrever uma relação de concentração de pessoas e oportunidades de emprego, conforme Bourdic, Salat e Nowacki (2012), sendo a densidade populacional dada pela equação 2 e a densidade de empregos, pela equação 3.

$$
\begin{aligned}
& \text { Densidade Populacional }=\frac{\text { população }}{\text { area de estudo }} \\
& \text { Densidade de empregos }=\frac{\text { total de emprego }}{\text { area de estudo }}
\end{aligned}
$$

Cruz e Marins (2017) e Willians et.al. (2000) apontam que as formas urbanas mais densas, mais diversificadas e mais compactas tendem a proporcionar um uso eficiente da terra. A compacidade é calculada de acordo com a comparação média entre o perímetro de cada distrito e o perímetro de um círculo que tem a mesma área, apontam Li e Yeh (2004), onde IC é o valor do índice de compacidade, $S_{j}$ e $P_{j}$ são a área e o perímetro do distrito analisado, dados pela equação 4. Os valores de IC mais baixos indicam um espaço mais disperso, segundo Lu e Liu (2016).

$$
\text { Indice de Compacidade }=I C=2 \times \frac{\sqrt{\pi \times S_{j}}}{P_{j}}
$$




\section{APLICAÇÃO DE MÉTRICAS SOCIOESPACIAIS URBANAS PARA CARACTERIZAÇÃO DAS DIVISÕES DO MUNICÍPIO DE SÃO PAULO}

São Paulo tem perímetro de $365 \mathrm{~km}$ e área de $1.520 \mathrm{~km}^{2}$, contando com 32 subdivisões. No entanto, as divisões de Sapopemba e Vila Prudente estão atualmente combinadas como uma só, formalizando 31 divisões, conforme figura 2.

Para aplicação de indicadores em áreas urbanas, esta pesquisa caracterizou as diferentes divisões administrativas do município de São Paulo, a partir da densidade populacional, da densidade de empregos, da diversidade de uso e da compacidade.

Figura 2 - Divisões Administrativas do Município de São Paulo

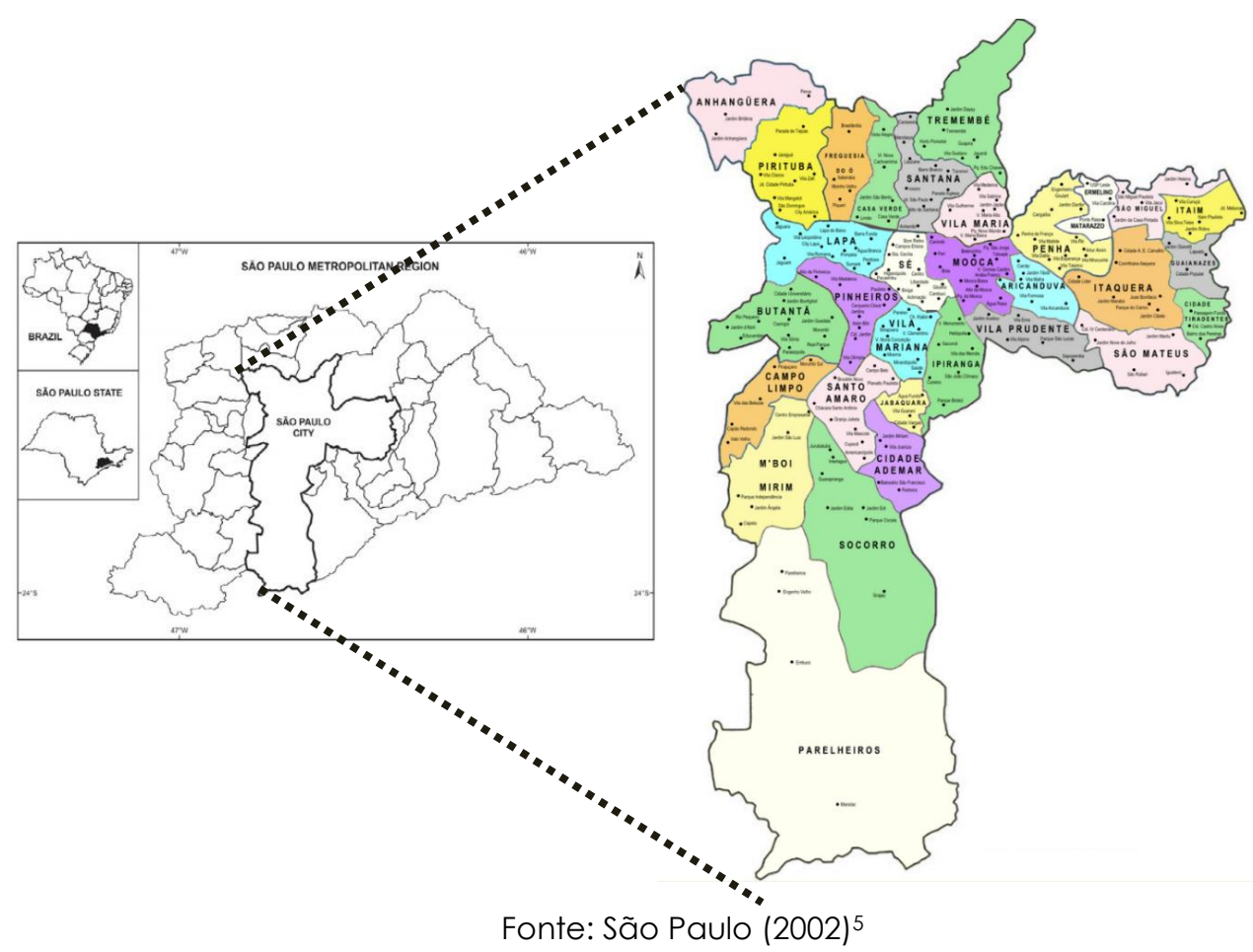

Foi estabelecida uma metodologia de agregação resultante do total de lotes destinados às categorias de uso, para cada propriedade cadastrada, gerando as 6 tipologias de uso apresentadas na tabela 1, para cada divisão administrativa do município.

O uso habitacional absorve todos os lotes destinados às habitações, sejam elas verticais ou horizontais, de qualquer padrão. O uso comercial reúne todos os lotes para atividades comerciais, escritórios e estacionamentos privados. $O$ uso industrial abrange todos os lotes com atividade industrial, armazéns e depósitos. O uso especial absorve hotéis, hospitais, e atividades da administração pública. O uso educacional envolve todos os estabelecimentos de ensino, de qualquer nível, das escolas primárias às universidades e centros de formação. O uso cultural reúne todos os lotes destinados a cinemas, teatros, centros culturais e templos religiosos.

\footnotetext{
5 São Paulo Lei 13.399/2002 - Prevê a criação das subprefeituras do município de São Paulo e outras providencias Disponível em:<http://www.prefeitura.sp.gov.br/cidade/upload/lei_13_399_1254940922.pdf> Acessado 29/03/2019
} 
Tabela 1 - Número total de Edificações por uso nas subdivisões administrativas do Munícipio de São Paulo

\begin{tabular}{|c|c|c|c|c|c|c|}
\hline Subdivisão & Habitacional & Comercial & Industrial & Especial & Educacional & Cultural \\
\hline Aricanduva & 6.598 & 663 & 18 & 11 & 9 & 59 \\
\hline Butantã & 9.555 & 1.172 & 46 & 13 & 31 & 81 \\
\hline Campo Limpo & 25.472 & 152 & 2 & 6 & 6 & 26 \\
\hline $\begin{array}{c}\text { Capela do } \\
\text { Socorro }\end{array}$ & 31.728 & 3.126 & 66 & 29 & 59 & 197 \\
\hline Casa Verde & 35.991 & 4.345 & 303 & 45 & 101 & 199 \\
\hline Cidade Ademar & 38.341 & 3.391 & 302 & 51 & 124 & 171 \\
\hline $\begin{array}{c}\text { Cidade } \\
\text { Tiradentes }\end{array}$ & 44.773 & 5.541 & 421 & 60 & 86 & 300 \\
\hline $\begin{array}{c}\text { Ermelino } \\
\text { Matarazzo }\end{array}$ & 45.283 & 6.263 & 164 & 59 & 92 & 288 \\
\hline Freguesia do Ó & 46.182 & 7.113 & 244 & 73 & 133 & 319 \\
\hline Guaianases & 49.236 & 5.688 & 424 & 70 & 146 & 257 \\
\hline Ipiranga & 49.328 & 10.824 & 270 & 60 & 138 & 343 \\
\hline Itaim Paulista & 55.844 & 5.779 & 553 & 106 & 161 & 185 \\
\hline Itaquera & 57.957 & 7.372 & 387 & 60 & 177 & 318 \\
\hline Jabaquara & 60.838 & 10.585 & 1.851 & 107 & 182 & 312 \\
\hline Lapa & 64.351 & 8.119 & 885 & 75 & 202 & 427 \\
\hline M'Boi Mirim & 73.902 & 6.744 & 503 & 81 & 188 & 291 \\
\hline Mooca & 75.388 & 9.022 & 1.302 & 232 & 204 & 322 \\
\hline Parelheiros & 92.374 & 6.686 & 472 & 115 & 163 & 401 \\
\hline Penha & 104.046 & 9.032 & 321 & 257 & 226 & 359 \\
\hline Perus & 104.355 & 10.543 & 1.252 & 102 & 179 & 409 \\
\hline Pinheiros & 105.330 & 16.668 & 942 & 2.256 & 384 & 284 \\
\hline Pirituba & 106.222 & 12.158 & 824 & 186 & 324 & 534 \\
\hline Santana & 115.762 & 12.464 & 324 & 1.208 & 328 & 345 \\
\hline Santo Amaro & 120.317 & 132.479 & 13.845 & 1.851 & 501 & 727 \\
\hline São Mateus & 122.026 & 9.333 & 509 & 164 & 300 & 329 \\
\hline São Miguel & 138.935 & 25.674 & 2.698 & 388 & 398 & 449 \\
\hline Sé & 154.615 & 22.428 & 1.690 & 415 & 416 & 439 \\
\hline Tremembé & 63.013 & 7.909 & 861 & 90 & 166 & 319 \\
\hline Vila Maria & 167.410 & 62.675 & 170 & 14.059 & 389 & 287 \\
\hline Vila Mariana & 199.705 & 33.358 & 288 & 3.072 & 716 & 425 \\
\hline Vila Prudente & 214.075 & 76.050 & 1.234 & 4.354 & 384 & 630 \\
\hline $\begin{array}{c}\text { Total } \\
\text { (São Paulo) }\end{array}$ & 2.578 .952 & 533.356 & 33.171 & 29.655 & 6.913 & 10.032 \\
\hline
\end{tabular}

Fonte: Os Autores (2019) Baseado em Dados Oficiais do Município de São Paulo

Os dados utilizados sobre o uso do solo urbano tiveram como fonte o Cadastro Territorial e Predial de Conservação e Limpeza (TPCL) 6 , mantido pelo Departamento de Arrecadação (DECAR), da Secretaria Municipal de Fazenda e Desenvolvimento Econômico da Prefeitura de São Paulo. Os dados populacionais ${ }^{7}$ são provenientes do Censo Demográfico 2010 , realizado pelo Instituto Brasileiro de Geografia e Estatística (IBGE) e pelos estudos e pesquisas da Fundação Sistema Estadual de Análise de Dados (SEADE) e estimativas da População, do

\footnotetext{
${ }^{6}$ É uma base de dados publica sob a responsabilidade a coordenação de produção e analise a informação (Geoinfo), vinculado à Secretaria Municipal de Urbanismo e Licenciamento (SMUL) de São Paulo. Foi utilizada a base de dados de 2015. Disponível em: https: //www.prefeitura.sp.gov.br/cidade/secretarias/urbanismo/dados_estatisticos/info_cidade/uso_do_solo_urbano/index.php $? p=260379$

7 Banco de dados públicos sob responsabilidade da Prefeitura do Município de São Paulo. Disponível em: https://www.prefeitura.sp.gov.br/cidade/secretarias/urbanismo/dados_estatisticos/info_cidade/demografia/index.php?p= 260265
} 
Departamento de Geografia e Estatística. Produção e Análise de Informações (Deinfo) da Secretaria Municipal de Desenvolvimento Urbano, gerando a tabela 2.

Tabela 2 - Dados Demográficos e Cartográficos das divisões administrativas do município de São Paulo

\begin{tabular}{|c|c|c|c|c|}
\hline Subdivisão & $\begin{array}{l}\text { Área } \\
\text { (ha) }\end{array}$ & $\begin{array}{c}\text { Perímetro } \\
\text { (m) }\end{array}$ & $\begin{array}{c}\text { População } \\
\text { (no habitantes) }\end{array}$ & $\begin{array}{c}\text { Empregos } \\
\text { (no empregos) }\end{array}$ \\
\hline Aricanduva & 2.233 & 22.573 & 267.702 & 87.398 \\
\hline Butantã & 5.642 & 41.056 & 428.217 & 182.244 \\
\hline Campo Limpo & 3.668 & 38.458 & 607.105 & 74.930 \\
\hline Capela do Socorro & 13.263 & 57.156 & 594.930 & 81.501 \\
\hline Casa Verde & 2.720 & 33.585 & 309.376 & 75.147 \\
\hline Cidade Ademar & 3.065 & 31.414 & 410.998 & 32.089 \\
\hline Cidade Tiradentes & 1.493 & 21.057 & 211.501 & 6.875 \\
\hline Ermelino Matarazzo & 1.598 & 22.509 & 207.509 & 23.782 \\
\hline Freguesia do ó & 3.210 & 30.607 & 407.245 & 50.084 \\
\hline Guaianases & 1.776 & 25.697 & 268.508 & 16.700 \\
\hline Ipiranga & 3.759 & 31.806 & 467.563 & 594.657 \\
\hline Itaim Paulista & 2.160 & 24.242 & 373.127 & 30.322 \\
\hline Itaquera & 5.509 & 38.728 & 523.848 & 75.647 \\
\hline Jabaquara & 1.403 & 19.360 & 223.780 & 71.111 \\
\hline Lapa & 4.057 & 37.998 & 305.526 & 392.136 \\
\hline M'Boi Mirim & 6.346 & 43.237 & 563.305 & 63.795 \\
\hline Mooca & 3.604 & 31.989 & 343.980 & 310.479 \\
\hline Parelheiros & 36.078 & 132.712 & 139.441 & 6.681 \\
\hline Penha & 4.335 & 39.425 & 474.659 & 91.443 \\
\hline Perus & 5.721 & 50.317 & 146.046 & 13.568 \\
\hline Pinheiros & 3.199 & 30.599 & 289.743 & 658.728 \\
\hline Pirituba & 5.534 & 38.309 & 437.592 & 66.723 \\
\hline Santana & 3.580 & 37.337 & 324.815 & 124.861 \\
\hline Santo Amaro & 3.776 & 32.278 & 238.025 & 316.872 \\
\hline São Mateus & 4.547 & 38.442 & 426.794 & 40.596 \\
\hline São Miguel & 2.605 & 40.013 & 369.496 & 34.038 \\
\hline Sé & 3.808 & 27.724 & 691.979 & 664.328 \\
\hline Tremembé & 6.530 & 49.023 & 291.867 & 41.227 \\
\hline vila Maria & 2.124 & 21.753 & 191.445 & 204.307 \\
\hline Vila Mariana & 2.625 & 21.901 & 344.067 & 194.947 \\
\hline Vila Prudente & 2.786 & 23.615 & 377.073 & 234.479 \\
\hline São Paulo & 152.753 & 365.000 & 11.257 .262 & 4.861 .695 \\
\hline
\end{tabular}

Fonte: Os Autores (2019) baseado em dados oficiais do município de São Paulo

Os dados cartográficos (área e perímetro), obtidos por meio do Portal GeoSampa ${ }^{8}$ e de imagens de satélite, obtidas do Instituto Nacional de Pesquisas Espaciais (INPE) ${ }^{9}$, foram utilizados. O mapa de São Paulo foi georreferenciado com as imagens do satélite CBERS-2 e foram medidos os perímetros e áreas de cada uma das 31 divisões do município de São Paulo, finalizando a tabela 2.

\footnotetext{
${ }^{8}$ GeoSampa é uma ferramenta de informações espaciais e geoprocessamento da Prefeitura do município de São Paulo. Disponível em: http://geosampa.prefeitura.sp.gov.br/PaginasPublicas/_SBC.aspx

${ }^{9}$ Instituto Nacional de Pesquisas Espaciais. Departamento de Geração de Imagens e Sensoriamento. São José dos Campos: INPE, 2015.
} 
Com base nos dados da pesquisa, foram calculadas a densidade populacional (hab /ha), a densidade de empregos (empregos /ha), o índice de diversidade de uso (I) e o índice de compacidade (IC) para as 31 divisões administrativas de São Paulo, na tabela 3, na qual a população é total de habitantes, e os empregos representam o número de oportunidades formais de trabalho10, em cada divisão.

Tabela 3 - Resultados para densidade populacional, Densidade de Empregos, índice de diversidade (I) e índice de compacidade para cada divisão administrativa do município de São Paulo

\begin{tabular}{|c|c|c|c|c|}
\hline Subdivisão & $\begin{array}{c}\text { Densidade Populacional } \\
\text { (hab/ha) }\end{array}$ & $\begin{array}{c}\text { Densidade de Empregos } \\
\text { (emp/ha) }\end{array}$ & $\begin{array}{l}1 \\
(-)\end{array}$ & $\begin{array}{l}C l \\
(-)\end{array}$ \\
\hline Aricanduva & 120 & 39 & 0,196 & 0,742 \\
\hline Butantã & 76 & 32 & 0,231 & 0,648 \\
\hline Campo Limpo & 166 & 20 & 0,145 & 0,558 \\
\hline Capela do Socorro & 45 & 6 & 0,188 & 0,714 \\
\hline Casa Verde & 114 & 28 & 0,229 & 0,550 \\
\hline Cidade Ademar & 134 & 10 & 0,182 & 0,625 \\
\hline Cidade Tiradentes & 142 & 5 & 0,235 & 0,650 \\
\hline Ermelino Matarazzo & 130 & 15 & 0,246 & 0,629 \\
\hline Freguesia do ó & 127 & 16 & 0,270 & 0,656 \\
\hline Guaianases & 151 & 9 & 0,222 & 0,581 \\
\hline Ipiranga & 124 & 158 & 0,345 & 0,683 \\
\hline Itaim Paulista & 173 & 14 & 0,205 & 0,679 \\
\hline Itaquera & 95 & 14 & 0,235 & 0,679 \\
\hline Jabaquara & 160 & 51 & 0,322 & 0,686 \\
\hline Lapa & 75 & 97 & 0,245 & 0,594 \\
\hline M'Boi Mirim & 89 & 10 & 0,182 & 0,653 \\
\hline Mooca & 95 & 86 & 0,240 & 0,665 \\
\hline Parelheiros & 4 & $<1$ & 0,150 & 0,507 \\
\hline Penha & 110 & 21 & 0,171 & 0,592 \\
\hline Perus & 26 & 2 & 0,202 & 0,533 \\
\hline Pinheiros & 91 & 206 & 0,300 & 0,655 \\
\hline Pirituba & 79 & 12 & 0,220 & 0,688 \\
\hline Santana & 91 & 35 & 0,212 & 0,568 \\
\hline Santo Amaro & 63 & 84 & 0,211 & 0,675 \\
\hline São Mateus & 94 & 9 & 0,154 & 0,622 \\
\hline São Miguel & 142 & 13 & 0,320 & 0,452 \\
\hline Sé & 182 & 174 & 0,262 & 0,789 \\
\hline Tremembé & 45 & 6 & 0,242 & 0,584 \\
\hline Vila Maria & 90 & 96 & 0,533 & 0,751 \\
\hline Vila Mariana & 131 & 74 & 0,293 & 0,829 \\
\hline Vila Prudente & 135 & 84 & 0,480 & 0,792 \\
\hline
\end{tabular}

Fonte: Os Autores (2019)

10 Baseado no Relatório anual de informações sociais (Rais). Disponível em: https: //www.Prefeitura.sp.gov.br/cidade/secretarias/urbanismo/dados_estatisticos/info_cidade/trabalho/index.php?p=260362 
Os resultado mostram na tabela 3, sob a perspectiva morfológica urbana, as cinco divisões com maiores índices de compacidade (e, portanto, mais compactas) são Vila Marina $(\mathrm{Cl}=$ $0,829)$, Vila Prudente $(I C=0,792)$, Sé $(I C=0,789)$. ), Vila Maria $(I C=0,751)$ e Aricanduva $(I C=$ 0,742 ). Por outro lado, as 5 divisões com maior índice de diversidade (e, portanto, mais diversificadas em termos de uso do solo) são Vila Maria $(I=0,533)$, Vila Prudente $(I=0,480)$, Ipiranga $(I=0,345)$, Jabaquara $(I=0,322)$ e São Miguel $(I=0,320)$.

Ao analisar os menores índices de compacidade, as subdivisões com menores índices (e, portanto, menos compactas) são São Miguel (IC =0,452), Parelheiros (IC =0,507), Perus (IC = $0,533)$, Casa Verde $(\mathrm{Cl}=0,550)$ e Campo Limpo $(\mathrm{Cl}=0,558)$. Por outro lado, as divisões menos diversificadas (com menores índices de diversidade) são Campo Limpo (I =0,145), Parelheiros $(0,150)$, São Mateus $(I=0,154)$, Penha $(I=0,171)$ e Cidade Ademar $(I=0,182)$.

Embora Vila Maria e Vila Prudente estejam na lista das cinco áreas mais compactas e diversificadas, as posições oscilaram. A única que manteve a posição foi Vila Prudente. Portanto, é difícil diferenciar se é uma coincidência, ou se não há relação direta, e dependente, da compactação com a diversidade.

As cinco divisões com maior densidade populacional são Sé (182 hab /ha), Itaim Paulista (173 hab/ha), Campo Limpo (166 hab/ha), Jabaquara (160 hab/ha) e Guainases (151 hab/ha). E as cinco divisões com menor densidade populacional são Parelheiros (4 hab /ha), Perus (26 hab/ha), Tremembé (45 hab/ha), Capela do Socorro (45 hab/ha) e Santo Amaro (63 hab/ha). As divisões de Pinheiros (206 empregos/ha), Sé (174 empregos/ha) e Ipiranga (158 empregos/ha), são aqueles com as maiores concentrações de empregos.

\section{CONCLUSÕES}

A divisão da Sé, uma região central, apresenta-se como uma das melhores quando analisadas simultaneamente a compactação, a densidade populacional e a densidade de postos de trabalho. Simultaneamente, a Vila Maria, uma região mais afastada do centro, também apresenta um dos melhores resultados quando se analisa a compacidade, a diversidade e a densidade de empregos.

Parelheiros e Campo Limpo são divisões que estão entre as menos compactas e menos diversificadas. Mas isso ainda não nos permite afirmar que existe uma relação direta e dependente entre compacidade e diversidade. Outras variáveis, não consideradas no presente estudo, podem influenciar de forma distinta, cada uma dessas métricas urbanas

Verificou-se que, de modo geral, a relação entre as métricas urbanas consideradas não é sistemática, ou seja, todos os melhores casos de compacidade, não estão associados aos melhores casos de diversidade, densidade humana e densidade de emprego. Dessa forma, não é possível se afirmar que há, necessariamente, uma dependência entre essas variáveis urbanas, quando analisados os dados consolidados das regiões administrativas do município de São Paulo, as quais, possivelmente, são influenciadas também por outros fatores urbanos.

\section{REFERÊNCIAS}

BOURDIC, L.; SALATA, S.; NOWACKI, C. Assessing cities: a new system of cross-scale spatial indicators, Building Research \& Information, v.40, n.5, 2012.pp. 592-605.

CRUZ, R.B.C.; MARINS, K.R.C. Avaliação do índice de compacidade nas subprefeituras do município de São Paulo - Revista Eletrônica De Engenharia Civil, v.13, n.2, 2017. pp. 287-298.

INSTITUTO BRASILEIRO DE GEORGRAFIA E ESTATISTICA (IBGE). Censo demográfico 2010. Características da população e dos domicílios: resultado do universo. Rio de Janeiro: IBGE, 2010.

MASERA, O.; ASTIER, M.; LÓPEZ-RIDAURA, S. Sustentabilidad y manejo de recursos naturales: el marco de evaluación MESMIS. México: Mundi Prensa, 2000, 109 p. 
LI, X.; YEH, A. G.O. Analyzing spatial restructuring of land use patterns in a fast-growing region using remote sensing and GIS. Landscape and Urban Planning, v.69, n.4, 2004. pp.335-354.

LU, C.; LIU, Y. Effects of China's urban form on urban air quality. Urban Studies, v.53, n.12, 2016. Pp. 2607-2623.

WILLIANS, K.; BURTON, E.; JENKS, M. Achieving Sustainable Urban Form. Londres. E \& FN. 408p. 2000.

ZHEN, F.; QIN, X.; YE, X.; SUNS, H., LUOSANG, Z. Analyzing urban development patterns based on the flow analysis method. Cities. Article in press, Octotber,2018. 